ORIGINAL ARTICLE

DOI: 10.34057/PPj.2020.39.03.002

Pelviperineology 2020;39(3):77-84

\title{
Anatomical outcomes of laparoscopic high uterosacral ligament suspension for primary apical prolapse
}

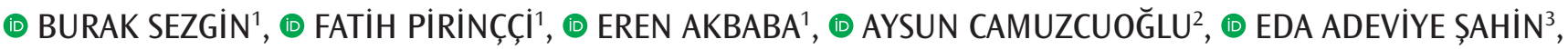 \\ (1) AHMET AKIN SIVASLIOĞLU1, (1) HAKAN CAMUZCUOĞLU ${ }^{4}$ \\ 1Department of Obstetrics and Gynaecology, Muğla Sıtkı Koçman University Faculty of Medicine, Muğla, Turkey \\ 2Department of Obstetrics and Gynaecology, Private Adatıp Sakarya Hospital, Sakarya, Turkey \\ ${ }^{3}$ Department of Obstetrics and Gynaecology, Malatya Training and Research Hospital, Malatya, Turkey \\ ${ }^{4}$ Private Clinic, Department of Gynaecologic Oncology, Sakarya, Turkey
}

\begin{abstract}
Objective: We aimed to evaluate the short-term anatomical results of laparoscopic high uterosacral ligament suspension (LHUSLS) in the surgical treatment of apical prolapse.

Materials and Methods: Medical records of thirty women with stage 2 or higher cuff or uterine apical prolapse who underwent LHUSLS operations were retrospectively analysed. Preoperative, intraoperative, and 6- and 12-month short-term postoperative data were recorded. Anatomical success was defined as no prolapse for any Pelvic Organ Prolapse Quantification (POP-Q) point at or below $1 \mathrm{~cm}$ above the hymen. To evaluate prolapse symptoms, we used the Pelvic Organ Prolapse Distress Inventory (POPDI)-6 questionnaire.

Results: Based on stage II of POP-Q as the recurrence criteria, the anatomical success rate was 100\%. The POP-Q point C, Aa, Ap, Ba and Bp measurements were significantly higher after surgery than the preoperative values. There was no recurrence or ureteral injury in any case. The scores of POPDI-6 before and after LHUSLS were significantly lower in all patients after surgery $(p<0.001)$.

Conclusion: LHUSLS is a simple and effective procedure that has been found to provide successful anatomical outcomes and reduced ureteral injury for apical prolapse. However, the long-term results of this procedure require further investigation.
\end{abstract}

Keywords: Apical prolapse; high uterosacral ligament suspension; laparoscopy; native tissue repair

\section{INTRODUCTION}

Pelvic organ prolapse (POP) is a clinical condition that affects approximately half of women who have given birth. ${ }^{1}$ The lifetime probability of a woman undergoing POP surgery was reported to be between 11 and $19 \%{ }^{2}$ Apical prolapse affects $11 \%$ of women. ${ }^{3}$ Currently, conservative and surgical treatment modalities are available for POP. POP repair can be performed vaginally or abdominally for patients who do not benefit from conservative methods or who require surgical treatment. Although mesh or natural tissue could be used during these interventions, the FDA recommendations regarding mesh usage have led many surgeons to negative thoughts regarding these methods. ${ }^{4}$ Therefore, alternative treatment modalities using native tissue are gaining popularity. Recent technological advances increase the preferability of minimally invasive approaches for both the surgeon and the patient.

Address for Correspondence: Burak Sezgin, Department of Obstetrics and Gynaecology, Muğla Sıtkı Koçman University Faculty of Medicine, Muğla, Turkey E-mail: buraksezgin@yahoo.com ORCID ID: orcid.org/0000-0003-2938-5816 Received: 31 October 2019 Accepted: 12 May 2020

(C) Copyright 2020 by the International Society for Pelviperineology / Pelviperineology published by Galenos Publishing House. 
Although the most common type of prolapse is anterior vaginal prolapse, the loss of apical support is typically present in prolapses below the hymen. The belief in providing adequate support to the vaginal apex for a durable and long-lasting surgical repair of patients with advanced stage prolapse is increasing. ${ }^{5}$ Anterior and posterior vaginal repairment may fail if adequate apex support is not provided, as the apex provides significant support to the vaginal wall.

Abdominal (laparoscopic/robotic) sacrocolpopexy is the gold standard method for apical prolapse treatment. Although this method has a high success rate, it has serious complications, such as nerve plexus and vascular damage. ${ }^{6}$ However, uterosacral ligament suspension (USLS) is a method that can be performed without mesh usage. According to the $\mathrm{FIGO}^{\circ}$ working group study, USLS can improve the vaginal apex to an anatomically better position than sacro spinous ligament fixation. Moreover, it has been reported that USLS has a high success rate and is a safe method (grade A recommendation).?

The uterosacral ligament is anatomically divided into three parts, including the proximal, intermediate and distal parts. The proximal part is the strongest part and is composed of connective tissue, fat and lymphatics. The distal part is often weak and damaged in POP patients. USLS has been shown to improve the apical compartment with a success rate of $98.3 \%{ }^{8}$ However, in USLS cases performed with a vaginal route, the ureter damage ratio may reach $11 \%$, but this rate may decrease to a lower level with a laparoscopic approach as better anatomic visualization can be achieved with laparoscopy. ${ }^{8}$

USLS was first described by Miller, and various modifications have been developed over time. Recently, a high uterosacral ligament suspension (HUSLS) technique has been developed by suturing the proximal part of the uterosacral ligament. ${ }^{9-11}$ This method is thought to provide a strong apical support to the vaginal cuff.

HUSLS is performed with native tissue without the use of mesh. Using this approach, mesh complications, which account for an important part of POP operation complications, can be eliminated. For this reason, we applied the laparoscopic high uterosacral ligament suspension (LHUSLS) method in our cases. In the literature, there are several video articles showing the LHUSLS surgical technique as a new and alternative method in the treatment of POP. However, we did not identify any study showing the effectiveness of this method in the postoperative period.

The aims of the study are to describe a new modification of LHUSLS and assess the efficacy of LHUSLS in the surgical treatment of apical prolapse.

\section{MATERIALS AND METHODS}

This retrospective case series study was approved by the Local Ethics Committee for Clinical Research of Muğla Sıtkı Koçman University, Faculty of Medicine, Muğla, Turkey (date: 07.02.19, decision no: 6). Between January 2015 and March 2018, data from patients who underwent LHUSLS for apical POPS (POP-Q 22) in our obstetrics and gyneacology clinics were retrospectively analysed. ${ }^{12}$ In the given period, only this technique was performed as the standard procedure for primary apical prolapse repairment in women with cuff prolapses or no demand of uterine preservation. The necessary information was obtained from the hospital database and patient files.

During this period, 45 patients underwent LHUSLS operation for apical prolapse by two surgeons with advanced laparoscopy experience. The data of 14 patients with a POP-Q stage value $\leq 1$, an abnormal pap smear and a suspected adnexial mass or malignancy were excluded from the study. The medical records of only one patient could not be found and were lost to follow up. Twenty-one women with uterine prolapse and nine women with vaginal cuff prolapse were included. Patient characteristics, such as age, gravidity, parity, body mass index (BMI), comorbidities (anaemia, diabetes, hypertension, and cardiac disease), menopausal status, hormone replacement therapy, tobacco usage, history of previous prolapse surgery, history of previous incontinence surgery, operation time, intraoperative complications, postoperative complications, and duration of hospital stay, were recorded from the hospital database. The records of the preoperative and $6^{\text {th }}$ and $12^{\text {th }}$ month postoperative POP-Q stages were also obtained from patient files. Anatomical success was defined as no prolapse of any POP-Q point at or below $1 \mathrm{~cm}$ above the hymen. The duration of hospitalization was calculated in days from the first postoperative day until the discharge time. Patient demographics were described. The preoperative and $6^{\text {th }}$ and $12^{\text {th }}$ month postoperative POP-Q stages were compared and analysed. Transvaginal ultrasonography scans, abdominal magnetic resonance imaging examinations (if performed) and Papanicolaou smear records were also analysed. The patients were grouped as uterine prolapse $(n=21)$ and cuff prolapse $(n=9)$. To evaluate prolapse symptoms, we used the Pelvic Organ Prolapse Distress Inventory (POPDI)-6 questionnaire. ${ }^{13}$ The Turkish validation of POPDI- 6 questionnaire was also performed. ${ }^{14}$

\section{Description of LHUSLS technique}

A standard laparoscopic hysterectomy is undertaken; nevertheless, bilateral uterosacral ligaments are not cut and preserved during hysterectomy. After the hysterectomy procedure, the vaginal 
cuff is closed intracorporeally with No: 0 absorbable polyglactin (MITSUTM, Meril Endo Surgery Private Limited, Muktanand Marg, Chala, Vapi 396191, Gujarat, India) in a single layer continuously. Then, in all cases, bilateral ureters are released by entering into the retroperitoneal space. The ureters are craniocaudally dissected and laterally shifted to the uterosacral ligaments. Using No: 2 polyglactin (MITSUTM, Meril Endo Surgery Private Limited, Muktanand Marg, Chala, Vapi 396191, Gujarat, India) in a circular fashion, we first sutured the proximal part of the uterosacral ligament, followed by the intermediate and distal parts, respectively. We subsequently stitch up two bites suture from the pubocervical fascia through the rectovaginal fascia and continue with the opposite uterosacral ligament. The distal, intermediate and proximal circular sutures are stitched up on the opposite side, respectively. Finally, two sutures in the proximal uterosacral ligaments are ligated together, and the suture is tightened (Figure 1). Moreover, an assistant checks the total vaginal length (TVL) transvaginally. The process is completed after ensuring that the desired suspension is achieved for the patient. In cases of cuff prolapse, an assistant helps to clarify the vaginal vault using the rectal anal dilator transvaginally. In this way, the bladder is initially dissected. The pubocervical fascia is then exposed anteriorly and the rectovaginal fascia posteriorly.

\section{Statistical Analysis}

Statistical analyses were performed using Statistical Package for the Social Sciences software, version 23 (SPSS, Inc., Chicago, IL). The data were expressed as the mean and range for continuous variables, and binary variables were reported as numbers and percentages. The Friedman's test, an alternative to the single factor variance analysis, was used for the not normally distributed data. A p value of less than 0.05 was accepted as statistically significant.

\section{RESULTS}

The baseline clinical and demographic characteristics of the patients are listed in Table 1. In particular, the mean BMI was $28 \pm 2.07 \mathrm{~kg} / \mathrm{m}^{2}$, and the mean age of the women was $54.6 \pm 4.48$ years. Eighty percent of the patients were postmenopausal. In regards to the preoperative $\mathrm{POP}-\mathrm{Q}$ stages, 10 patients were in POP-Q stage 4, 15 patients were in POP-Q stage 3 and five patients were in POP-Q stage 2. None of the patients had previous uterine prolapse surgery. A history of previous incontinence surgery was found in $16.6 \%$ of the patients. No hormone replacement therapy was identified in the patients. Tobacco usage was reported as $23.3 \%$. Medical comorbidities were reported as 50\%

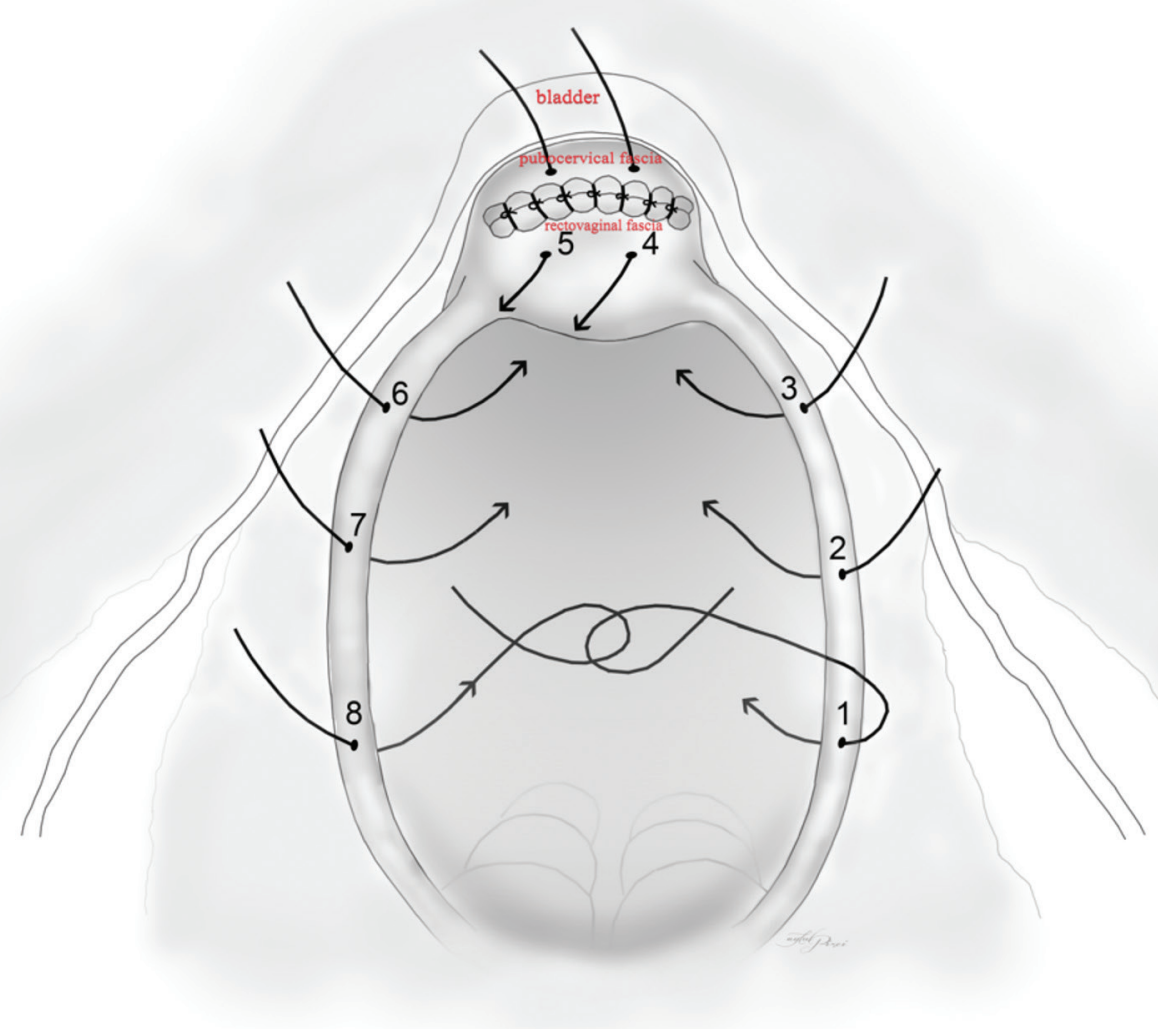

Figure 1. Description of LHUSLS technique

LHUSLS: Laparoscopic high uterosacral ligament suspension 


\begin{tabular}{|l|l|}
\hline \multicolumn{2}{|l|}{ Table 1. Baseline characteristics of patients ( $\mathbf{n}=30)$} \\
\hline Age & Mean \pm SD \\
\hline Gravid & $54.6 \pm 4.48$ \\
\hline Parity & $4.13 \pm 1.70$ \\
\hline BMI & $2.93 \pm 1.05$ \\
\hline Menopausal status (\%) & $28 \pm 2.07$ \\
\hline Postmenopausal & \multicolumn{2}{|l|}{} \\
\hline Premenopausal & 80 \\
\hline Tobacco usage (\%) & 20 \\
\hline Previous incontinence surgery (\%) & 23.3 \\
\hline Comorbidities (\%) & 16.6 \\
\hline No comorbidities & \multicolumn{2}{|l|}{} \\
\hline Anaemia & 50 \\
\hline Cardiac disease & 20 \\
\hline Hypertension & 6.7 \\
\hline Diabetes & 16.7 \\
\hline Preop POP-Q stage (n=30) & 6.7 \\
\hline Stage 1 & $10 / 30$ \\
\hline Stage 2 & 0 \\
\hline Stage 3 & $5 / 30$ \\
\hline Stage 4 & $15 / 30$ \\
\hline $\begin{array}{l}\text { BMl: Body mass index, POP-Q: Pelvic Organ Prolapse Quantification, SD: } \\
\text { Standard deviation, n: Number } \\
\text { Data are presented as mean } \pm \text { standard deviation or number (\%) }\end{array}$ \\
\hline
\end{tabular}

in the patients (anaemia 20\%, cardiac disease 6.7\%, hypertension $16.7 \%$, and diabetes $6.7 \%$ ).

All patients were followed up at the $6^{\text {th }}$ and $12^{\text {th }}$ months. Based on stage II of POP-Q as the recurrence criteria, the anatomical success rate was 100\% (30/30) for both 6 and 12 months. We did not identify recurrence in any patient. The POP-Q point C, Aa, Ap, Ba and Bp measurements were significantly higher at the $6^{\text {th }}$ and $12^{\text {th }}$ months after surgery than those at the preoperative time point in both groups $(\mathrm{p}<0.05)$ (Table 2A and 2B) (Figure 2A and $2 B)$. However, no significant difference was identified between the $6^{\text {th }}$ and $12^{\text {th }}$ months postoperatively. In both groups, there was no significant difference between the preoperative and postoperative measurements of the TVL $(p>0.05)$. The mean improvement for the Aa, Ap, Ba and Bp points was $2.9 \mathrm{~cm}, 2.09$ $\mathrm{cm}, 3.38 \mathrm{~cm}$ and $2.81 \mathrm{~cm}$, respectively, in the uterine prolapse group at 12 months postoperatively. The mean improvement for the Aa, Ap, Ba and Bp points was $2.77 \mathrm{~cm}, 2.22 \mathrm{~cm}, 3.45 \mathrm{~cm}$ and $2.78 \mathrm{~cm}$, respectively, in the cuff prolapse group at 12 months postoperatively. Preoperative and postoperative 12 months of POPDI-6 questionnaire results were presented at Table 3.

No cases of intraoperative complications, such as urinary tract injury, bowel/intestinal injury, or intraoperative blood transfusion due to massive haemorrhage, were reported. The mean operative duration time of the cuff prolapses group and the uterine prolapses group was $93.88 \pm 6.97$ minutes and $119.04 \pm 6.82$ minutes, respectively. No case of postoperative

Table 2A. Preoperative and postoperative POP-Q measurements in uterine prolapse group, $(n=21)$

\begin{tabular}{|c|c|c|c|c|c|c|c|c|c|}
\hline & & $\mathrm{Aa}$ & $\mathrm{Ba}$ & C & Ap & Bp & Gh & $\mathbf{P b}$ & TVL \\
\hline \multirow{4}{*}{ Pre } & Mean (SD) & $0.76(1.34)$ & $1.05(2.20)$ & $3.33(2.31)$ & $0.33(1.15)$ & $0.62(2.14)$ & $4.74(0.70)$ & $2.40(0.78)$ & $8.14(1.12)$ \\
\hline & Median & 1 & 1 & 3 & 0.0 & 0.0 & 5 & 2.5 & 8 \\
\hline & Range & 5 & 10 & 8 & 5 & 10 & 2.5 & 2.5 & 4 \\
\hline & Mean rank & $2.95^{\text {aa }}$ & $2.98^{\mathrm{ac}}$ & $3^{\text {af }}$ & $2.79^{\text {ah }}$ & $2.88^{\mathrm{ak}}$ & $2.36^{\text {aab }}$ & $2.17^{\text {aba }}$ & 2.05 \\
\hline \multirow{4}{*}{ P0P-Q6 } & Mean (SD) & $-2.14(0.96)$ & $-2.52(1.08)$ & $-7.48(0.81)$ & $-1.90(0.89)$ & $-2.24(0.77)$ & $4.5(0.71)$ & $2.33(0.73)$ & 8.05 (1.08) \\
\hline & Median & -2 & -3 & -8 & -2 & -2 & 4.5 & 2.5 & 8 \\
\hline & Range & 3 & 4 & 3 & 3 & 2 & 3 & 2.5 & 4 \\
\hline & Mean rank & $1.52^{\mathrm{ab}}$ & $1.40^{\mathrm{ad}}$ & $1.38^{\mathrm{ae}}$ & $1.50^{\mathrm{ag}}$ & $1.52^{\mathrm{al}}$ & $1.90^{\mathrm{aab}}$ & $1.95^{\text {aba }}$ & 1.95 \\
\hline \multirow{4}{*}{ P0P-Q12 } & Mean (SD) & $-2.14(1.01)$ & $-2.33(1.06)$ & $-7.29(0.94)$ & $-1.76(0.99)$ & $-2.19(0.93)$ & $4.43(0.78)$ & $2.31(0.72)$ & $8.10(1.06)$ \\
\hline & Median & -2 & -3 & -7 & -2 & -2 & 4.5 & 2.5 & 8 \\
\hline & Range & 3 & 4 & 3.5 & 3 & 3 & 3 & 2.5 & 4 \\
\hline & Mean rank & $1.52^{\mathrm{ab}}$ & $1.62^{\mathrm{ad}}$ & $1.62^{\mathrm{ae}}$ & $1.71^{\mathrm{ag}}$ & $1.60^{\mathrm{al}}$ & $1.74^{\mathrm{dab}}$ & $1.88^{\mathrm{dba}}$ & 2 \\
\hline \multirow{2}{*}{$\begin{array}{l}\text { Pre } \\
- \\
\text { P0P-Q6 } \\
- \\
\text { P0P-Q12 }\end{array}$} & Chi-square & 37.5 & 38.82 & 39.65 & 27 & 33.19 & 13.92 & 6.5 & 0.5 \\
\hline & $p$ value & $<0.05^{*}$ & $<0.05^{*}$ & $<0.05^{*}$ & $<0.05^{*}$ & $<0.05^{*}$ & $<0.05^{*}$ & $<0.05^{*}$ & $>0.05$ \\
\hline
\end{tabular}


Table 2B. Preoperative and postoperative POP-Q measurements in cuff prolapse group $(n=9)$

\begin{tabular}{|c|c|c|c|c|c|c|c|c|c|}
\hline & & $\mathrm{Aa}$ & $\mathrm{Ba}$ & C & Ap & Bp & Gh & $\mathrm{Pb}$ & TVL \\
\hline \multirow{4}{*}{ Pre } & Mean (SD) & $1.33(1.12)$ & $1.67(1.22)$ & $5.67(1.66)$ & $0.89(1.27)$ & $1(1.58)$ & $5.22(0.83)$ & $2.33(0.87)$ & $8(1.41)$ \\
\hline & Median & 1 & 2 & 6 & 1 & 1 & 5 & 2.5 & 8 \\
\hline & Range & 4 & 4 & 5 & 4 & 4 & 2 & 2.5 & 4 \\
\hline & Mean rank & $2.78^{\mathrm{aa}}$ & $2.78^{\mathrm{ac}}$ & $3^{\text {af }}$ & $2.78^{\mathrm{ah}}$ & $2.89^{\mathrm{ak}}$ & $2.67^{\mathrm{aab}}$ & 2.28 & 2 \\
\hline \multirow{4}{*}{ P0P-Q6 } & Mean (SD) & $-1.67(1.5)$ & $-1.89(1.54)$ & $-7.67(1.12)$ & $-1.11(0.78)$ & $-1.78(0.67)$ & $4.83(0.56)$ & $2.22(0.79)$ & $8(1.22)$ \\
\hline & Median & -2 & -2 & -7 & -1 & -2 & 5 & 2.5 & 8 \\
\hline & Range & 5 & 5 & 3 & 2 & 2 & 1.5 & 2 & 4 \\
\hline & Mean rank & $1.50^{\mathrm{ab}}$ & $1.56^{\mathrm{ad}}$ & $1.44^{\mathrm{ae}}$ & $1.67^{\mathrm{ag}}$ & $1.56^{\mathrm{al}}$ & $1.83^{\mathrm{aab}}$ & 1.78 & 2 \\
\hline \multirow{4}{*}{ P0P-Q12 } & Mean (SD) & $-1.44(1.13)$ & $-1.78(1.48)$ & $-7.56(1.24)$ & $-1.33(0.87)$ & $-1.78(0.67)$ & $4.72(0.67)$ & $2.17(0.79)$ & $8(1.22)$ \\
\hline & Median & -2 & -2 & -7 & -2 & -2 & 5 & 2 & 8 \\
\hline & Range & 4 & 5 & 3 & 2 & 2 & 2 & 2 & 4 \\
\hline & Mean rank & $1.72^{\mathrm{ab}}$ & $1.67^{\mathrm{ad}}$ & $1.56^{\mathrm{ae}}$ & $1.56^{\mathrm{ag}}$ & $1.56^{\mathrm{al}}$ & $1.50^{\mathrm{dab}}$ & 1.94 & 2 \\
\hline \multirow{2}{*}{$\begin{array}{l}\text { Pre } \\
- \\
\text { P0P-Q6 } \\
\text { - } \\
\text { POP-Q12 }\end{array}$} & Chi-square & 9.74 & 10.57 & 17.43 & 9.87 & 16 & 11.14 & 4.67 & 0.0 \\
\hline & p value & $<0.05^{*}$ & $<0.05^{*}$ & $<0.05^{*}$ & $<0.05^{*}$ & $<0.05^{*}$ & $<0.05^{*}$ & $>0.05$ & $>0.05$ \\
\hline
\end{tabular}

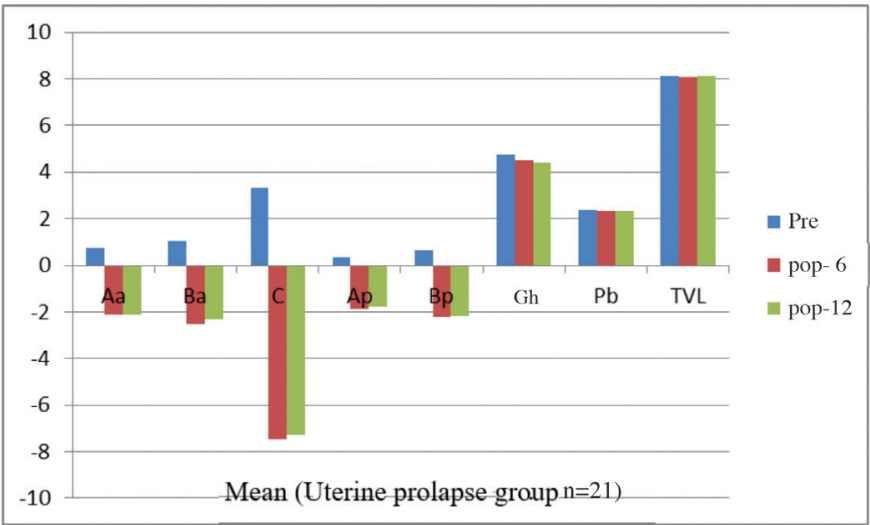

Figure 2A. Anatomical outcomes according to POP-Q points in Uterine prolapse group

POP-Q: Pelvic Organ Prolapse Quantification, Gh: Genital hiatus, Pb: Perineal body, TVL: Total vaginal length, n: Number

complications, such as haemorrhage, need for blood transfusion or fever was reported. The postoperative hospital stay was 2 days.

\section{DISCUSSION}

This is the first report to describe a case series study of LHUSLS performed with our technique. In this study, we aimed to demonstrate the efficacy and the short-term results of our LHUSLS technique in the treatment of apical prolapse. In the 12-month follow-up period, we obtained high success rates in all apical, anterior and posterior compartments. Furthermore, we obtained low recurrence and complication rates.

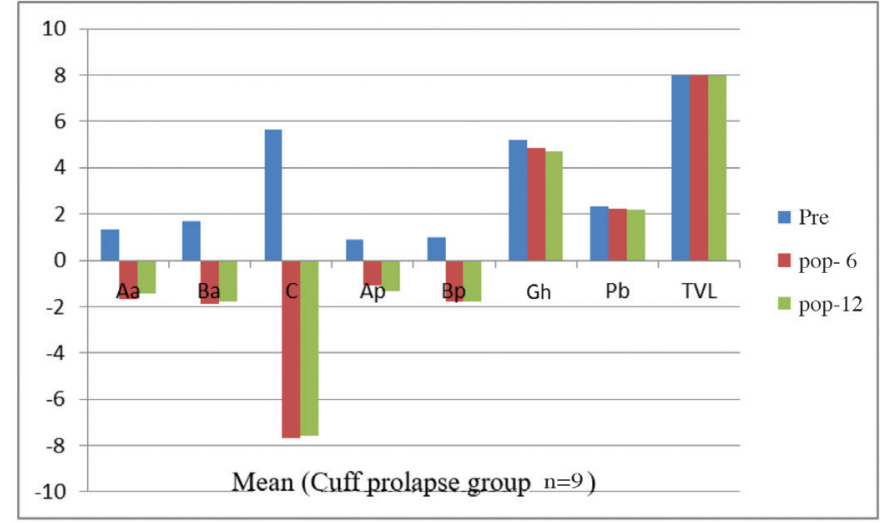

Figure 2B. Anatomical outcomes according to POP-Q points in Cuff prolapse group

POP-Q: Pelvic Organ Prolapse Quantification, Gh: Genital hiatus, Pb: Perineal body, TVL: Total vaginal length, $n$ : Number

\section{Table 3. Pre- and postoperative POPDI- 6 data}

\begin{tabular}{|l|l|l|l|}
\hline Questionnaire & Before $(\mathbf{n}=30)$ & After $(\mathbf{n}=30)$ & $\mathbf{p}$ \\
\hline POPDI-6 & $74.55 \pm 13.63$ & $21.91 \pm 9.90$ & $<0.001$ \\
\hline
\end{tabular}

POPDI-6: Pelvic Organ Prolapse Distress Inventory, n: Number, SD:

Standard deviation

Values are presented as mean score \pm SD

In the study of Sun et al. ${ }^{15}$, they performed LHUSLS with cervical amputation in 34 patients and achieved a 100\% anatomical success rate as in our study. In the study of Smith et al. ${ }^{16}$, they compared vaginal HUSLS and robotic sacrocolpopexy (RSC) 
in the long term, and they found that HUSLS was as effective as RSC (93.2\% for USLS vs $91.3 \%$ for RSC). They also reported a mesh erosion rate of $3.2 \%$ in the RSC group. Nishimura et al. ${ }^{17}$ evaluated perioperative complications and the anatomical outcomes of laparoscopic uterosacral colpopexy in 152 uterine prolapse patients. They reported a $19 \%$ of total recurrence rate. In our study, we also found an anatomical success rate of $100 \%$ using native tissue in the 12-month follow up period. We believe that apical suspension procedures using native tissue will gain more popularity following the mesh related recommendations of the FDA.

Dallas et al. ${ }^{18}$ suggested that hysterectomy should be performed in patients undergoing prolapse surgery in a large populationbased cohort study and found that the risk of prolapse surgery in the future is reduced by $1-3 \%$ using this approach. We also performed apical suspension together with hysterectomy in patients who did not demand uterine preservation. We did not have a recurrence surgery during the 12-month follow up period. Milnerowicz-Nabzdyk and Zimmer ${ }^{19}$ described the technique of laparoscopic trans-teres vault suspension and suspended the vaginal stump to the anterior rectus fascia in eight patients. Although they obtained anatomically pleasing results, fibular nerve damage developed in one of the eight patients. They also performed anterior and posterior vaginal repairment in addition to standard procedures in patients with an advanced stage. Although alternative pop treatment options using different anatomic structures are offered, such as in Milnerowicz's study, the complications are not clearly established. In our study, no complication was observed in any of our patients, and we did not perform an additional surgical procedure other than LHUSLS even in patients with stage 4 prolapse. In our study, we achieved satisfactory improvements in both the anterior and posterior compartments after adequate apical suspension.

In a retrospective study by Haj Yahya et al. ${ }^{20}, 48$ patients had hysteropexy with LUSLS and anterior colporrhaphy for anterior and apical prolapse. They reported an $85,4 \%$ anatomical success rate. In contrast to our study, they fixed the pubocervical fascia to the pericervical ring during anterior colporrhaphy. As indicated in their study, they maintained the continuity of strong supporting structures, such as the pubocervical fascia and uterosacral ligament. We also believe that the continuity of strong supporting structures is critical. However, we employed a different approach to ensure the direct continuity of the uterosacral ligament and pubocervical ligament. We suggest that the reason why the HUSLS applied in our practice is more effective may be the direct continuity of the pubocervical fascia and uterosacral ligament, which was provided by laparoscopic suturing.
It has been reported that intraperitoneal USLS techniques performed by a laparoscopical or transvaginal route have a high rate of ureteric injury, between 0 and 10.9\%. ${ }^{21}$ Karmakar et al. ${ }^{22}$ reported three ureteral injuries in the first 100 cases of extraperitoneal vaginal HUSLS, and they had to open the sutures. In the study of Houlihan et al. ${ }^{23}$, they did not report any ureteral damage in 54 cases of laparoscopic USLS as in our study. One of the main advantages of the laparoscopy is the improved visualization of the anatomic structures. In addition, because of the close proximity of the proximal part of the uterosacral ligament and the ureter, we dissected and lateralized the ureters. Using this approach, the possibility of ureter damage was minimized. In the Karmakar et al. ${ }^{22}$ study, while the anatomic success rate was $76 \%$, the recurrence rate requiring surgery was reported to be $14 \%$. Houlihan et al. ${ }^{23}$ found the recurrent prolapse rate to be lower in the laparoscopic group than in the vaginal group, at $4 \%$ and $15 \%$, respectively. In our study, the anatomic success rate was $100 \%$ in the 12 -month period and there was no recurrence. Thus, we suggest that the intraperitoneal approach can provide better results in terms of both ureteral injury and anatomical success than an extraperitoneal approach in the early period. However, it is clear that long-term results are required.

Milani et al. ${ }^{24}$ sutured the intermediate section of the uterosacral ligament in their study of transvaginal HUSLS. They reported a 2.6\% ureteral kinking rate and a $13.7 \%$ total recurrence rate. In our laparoscopic approach study, the sutures were first placed in the proximal part where the uterosacral ligament is the strongest. Furthermore, after completion of the suspension procedure, the degree of suspension can be viewed and evaluated directly. It is also possible to provide suspension support with extra sutures to the uterosacral ligament if necessary. Although the followup period of our study was limited to 12 months, it has been suggested that the laparoscopic approach may be more effective than the transvaginal approach in terms of ureteral injury and recurrence rates in the short term.

Turner et al. ${ }^{25}$ compared laparoscopic and transvaginal USLS ligament suspension and found that both methods had similar anatomical results. In their study, the median follow-up time was 21.5 weeks, and the median TVL was significantly longer in the laparoscopic group than it was in the vaginal group $(8.3 \pm 1.1$ $\mathrm{cm}$ vs $7.4 \pm 1.2 \mathrm{~cm}$ ). Similarly, in our study, the TVL was $8.10 \mathrm{~cm}$ and $8 \mathrm{~cm}$ in the uterine and cuff prolapse patients, respectively, at the $12^{\text {th }}$ month follow up. In the same study, they performed preoperative ureteral stenting in all cases with USLS. Nevertheless, the ureteral injury rate was $1.9 \%$ in their laparoscopic USLS cases. According to these data, we suggest that preoperative ureteral stent insertion might reduce but not eliminate ureteral injury risk. Although we did not insert a ureteral stent in any 
of our cases, we did not experience any ureteral injury. We want to emphasize that to prevent or minimize ureteral injury, bilateral ureteral releasing, lateralizing and suturing after visualization of the bilateral ureters is the most important step of our modification. Furthermore, according to our experience, the presence of a stent in the ureter during laparoscopy may reduce the ureter flexibility, which may thus complicate ureter dissection.

The limitations of our study included the low number of patients, the short follow-up period, and the retrospective nature. To generalize the results of this study, randomized controlled studies with larger patient groups and longer follow-up periods are required. The positive aspects of our study included the standardization of the follow-up periods and patient symptom assessment by a validated questionnaire. Although our study lacks the advantages of prospective studies, we believe that our preliminary data will guide future studies.

\section{CONCLUSIONS}

In conclusion, LHUSLS is a simple and effective procedure that has been found to provide successful anatomical outcomes and reduce ureteral injury for apical prolapse. However, in addition to this knowledge of ureteral releasing, lateralizing and uterosacral ligament suturing after visualization of the ureters with the advantage of laparoscopy, the anatomical results may be improved and complications may be minimized with this presented technique.

\section{ACKNOWLEDGMENTS}

The authors thank to Dr. Gülin YEĞiN for her assistance on statistical analysis.

\section{ETHICS}

Ethics Committee Approval: This retrospective case series study was approved by the Local Ethics Committee for Clinical Research of Muğla Sıtkı Koçman University, Faculty of Medicine, Muğla, Turkey (date: 07.02.19, decision no: 6).

Informed Consent: Informed patient consent was obtained.

Peer-review: Externally peer-reviewed.

\section{DISCLOSURES}

Conflict of Interest: There was no conflict of interest.

Financial Disclosure: The authors of the publication did not receive any financial support by any grant/research sponsor.

\section{REFERENCES}

1. Cruikshank SH, Kovac SR. Randomized comparison of three surgical methods used at the time of vaginal hysterectomy to prevent posterior enterocele. Am J Obstet Gynecol 1999; 180: 859-65.
2. Smith FJ, Holman CDJ, Moorin RE, Tsokos N. Lifetime risk of undergoing surgery for pelvic organ prolapse. Obstet Gynecol 2010; 116: 1096-100.

3. Samuelsson EC, Victor FT, Tibblin G, Svärdsudd KF. Signs of genital prolapse in a Swedish population of women 20 to 59 years of age and possible related factors. Am J Obstet Gynecol 1999; 180: 299305.

4. Skoczylas LC, Turner LC, Wang L, Winger DG, Shepherd JP. Changes in prolapse surgery trends relative to FDA notifications regarding vaginal mesh. Int Urogynecol J 2014; 25: 471-7.

5. Maher C, Feiner B, Baessler K, Christmann-Schmid C, Haya N, Brown J. Surgery for women with apical vaginal prolapse. Cochrane Database Syst Rev 2016; 10: CD012376.

6. Parkes IL, Shveiky D. Sacrocolpopexy for treatment of vaginal apical prolapse: evidence-based surgery. J Minim Invasive Gynecol 2014; 21: 546-57.

7. Betschart C, Cervigni M, Contreras Ortiz O, et al. Management of apical compartment prolapse (uterine and vault prolapse): A FIGO Working Group report. Neurourol Urodyn 2017; 36: 507-13.

8. Margulies RU, Rogers MAM, Morgan DM. Outcomes of transvaginal uterosacral ligament suspension: systematic review and metaanalysis. Am J Obstet Gynecol 2010; 202: 124-34.

9. Restaino S, Ronsini C, Finelli A, Santarelli A, Scambia G, Fanfani F. Laparoscopic Approach for Shull Repair of Pelvic Floor Defects. J Minim Invasive Gynecol 2018; 25: 954.

10. Panico G, Campagna G, Caramazza D, et al. Laparoscopic high uterosacral ligament suspension: an alternative route for a traditional technique. Int Urogynecol J 2018; 29: 1227-9.

11. Jan H, Ghai V, Doumouchtsis SK. Mesh-Free Laparoscopic High Uterosacral Ligament Suspension during Total Laparoscopic Hysterectomy for Uterine Prolapse. J Minim Invasive Gynecol 2018; 25: 952-3.

12. Bump RC, Mattiasson A, Bø K, et al. The standardization of terminology of female pelvic organ prolapse and pelvic floor dysfunction. Am J Obstet Gynecol 1996; 175: 10-17.

13. Barber MD, Walters MD, Bump RC. Short forms of two conditionspecific quality-of-life questionnaires for women with pelvic floor disorders (PFDI-20 and PFIQ-7). Am J Obstet Gynecol 2005; 193: 10313.

14. Toprak Celenay S, Akbayrak T, Kaya S, Ekici G, Beksac S. Validity and reliability of the Turkish version of the Pelvic Floor Distress Inventory-20. Int Urogynecol J 2012; 23: 1123-7.

15. Sun Z, Zhu L, Hu H, Lang J, Shi H, Gong X. [Laparoscopic high uterosacral ligament suspension combined with cervical amputation in treatment of women severe uterine prolapsed at child-bearing period]. Zhonghua Fu Chan Ke Za Zhi 2014; 49: 167-71.

16. Smith BC, Crisp CC, Kleeman SD, Yook E, Pauls RN. Uterosacral Ligament Suspension Versus Robotic Sacrocolpopexy for Treatment of Apical Pelvic Organ Prolapse. Female Pelvic Med Reconstr Surg 2019; 25: 93-8.

17. Nishimura K, Yoshimura K, Hoshino K, Myoga M, Kubo T, Hachisuga T. Laparoscopic uterosacral ligament colpopexy for apical support in addition to hysterectomy for pelvic organ prolapse. J Obstet Gynaecol Res 2019: 45: 1522-9. 
18. Dallas K, Elliott CS, Syan R, Sohlberg E, Enemchukwu E, Rogo-Gupta L. Association Between Concomitant Hysterectomy and Repeat Surgery for Pelvic Organ Prolapse Repair in a Cohort of Nearly 100,000 Women. Obstet Gynecol 2018; 132: 1328-36.

19. Milnerowicz-Nabzdyk E, Zimmer M. Laparoscopic trans teres vault suspension, a new laparoscopic method of treatment of female genital prolapse - a preliminary report. Prz menopauzalny = Menopause Rev 2016; 15: 6-11.

20. Haj Yahya R, Chill HH, Herzberg S, Asfour A, Lesser S, Shveiky D. Anatomical Outcome and Patient Satisfaction After Laparoscopic Uterosacral Ligament Hysteropexy for Anterior and Apical Prolapse. Female Pelvic Med Reconstr Surg 2018; 24: 352-5.

21. Shull BL, Bachofen C, Coates KW, Kuehl TJ. A transvaginal approach to repair of apical and other associated sites of pelvic organ prolapse with uterosacral ligaments. Am J Obstet Gynecol 2000; 183: 136573.
22. Karmakar D, Dwyer PL, Thomas E, Schierlitz L. Extraperitoneal uterosacral suspension technique for post hysterectomy apical prolapse in 472 women: results from a longitudinal clinical study. BJOG 2019; 126: 536-42.

23. Houlihan S, Kim-Fine S, Birch C, Tang S, Brennand EA. Uterosacral vault suspension (USLS) at the time of hysterectomy: laparoscopic versus vaginal approach. Int Urogynecol J 2019; 30: 611-21.

24. Milani R, Frigerio M, Cola A, Beretta C, Spelzini F, Manodoro S. Outcomes of Transvaginal High Uterosacral Ligaments Suspension. Female Pelvic Med Reconstr Surg. 2018;24:203-6.

25. Turner LC, Lavelle ES, Shepherd JP. Comparison of complications and prolapse recurrence between laparoscopic and vaginal uterosacral ligament suspension for the treatment of vaginal prolapse. Int Urogynecol J 2016; 27: 797-803. 